\title{
Relation of E1 pygmy and toroidal resonances
}

\author{
V.O. Nesterenko ${ }^{1, a}$, A. Repko², P.-G. Reinhard ${ }^{3}$, and J. Kvasil ${ }^{2}$ \\ ${ }^{1}$ Laboratory of Theoretical Physics, Joint Institute for Nuclear Research, Dubna, Moscow region, 141980, Russia \\ ${ }^{2}$ Institute of Particle and Nuclear Physics, Charles University, CZ-18000, Praha 8, Czech Republic \\ ${ }^{3}$ Institut für Theoretische Physik II, Universität Erlangen, D-91058, Erlangen, Germany
}

\begin{abstract}
A possible relation of low-lying E1 strength ("pygmy resonance") and toroidal strength is analyzed by using Skyrme random-phase-approximation (RPA) results for the strength functions, transition densities and current fields in ${ }^{208} \mathrm{~Pb}$. It is shown that the irrotational pygmy motion can appear as a local manifestation of the collective vortical toroidal dipole resonance (TDR) at the nuclear surface. The RPA results are compared to unperturbed (1ph) ones.
\end{abstract}

\section{Introduction}

Last years there is a high interest in low-lying E1 strength, often called pygmy dipole resonance (PDR) $[1,2]$. The PDR strength is related to nuclear matter properties (symmetry energy, incompressibility, effective masses) [3] and so can deliver useful complementing information for building the isospin-dependent part of the nuclear equation of state and for astrophysical applications.

The PDR is fragmented into several peaks [3] which can be separated into isoscalar $(\mathrm{T}=0)$ low-energy and isospin-mixed $(\mathrm{T}=0,1)$ high-energy parts [4]. In our recent study [5], we suggested that the low-energy isoscalar part of the PDR can be interpreted as a peripheral fraction of the isoscalar toroidal dipole resonance (TDR) $[6,7]$. The statement was based on a careful analysis of the E1 $(\mathrm{T}=0)$ strength functions, transition densities and current fields in ${ }^{208} \mathrm{~Pb}$, performed within the Skyrme random-phaseapproximation (RPA).

This suggestion is not so obvious if one views the PDR as a collective mode built from oscillations of the neutron excess against the nuclear core $\mathrm{N}=\mathrm{Z}$ [1], i.e. as a fully irrotational mode occurring in nuclei with $\mathrm{N}>\mathrm{Z}$. Instead, the TDR is a vortical motion [5,6]. Moreover, the TDR seems to be the main carrier of the nuclear dipole vorticity [8]. This resonance should exist in all nuclei independent of its neutron content. After extraction of the center-of-mass corrections $(\mathrm{cmc})$, the TDR dominates in low-energy E1 $(\mathrm{T}=0)$ excitations and, probably, constitutes the low-energy part of the isoscalar giant dipole resonance (ISGDR) observed in $\left(\alpha, \alpha^{\prime}\right)$ reaction [9]. The TDR transition operator is [10]

$$
\hat{M}_{\mathrm{TDR}}(E 1 \mu) \propto \int d^{3} r\left[r^{3}-\frac{5}{3} r\left\langle r^{2}\right\rangle_{0}\right] \vec{Y}_{11 \mu}(\hat{\vec{r}}) \cdot[\vec{\nabla} \times \hat{\vec{j}}(\vec{r})],
$$

\footnotetext{
a e-mail: nester@theor.jinr.ru
}

where $\hat{\vec{j}}(\vec{r})$ is the operator of the nuclear current, $\vec{Y}_{11 \mu}(\hat{\vec{r}})$ is the vector spherical harmonic. The cmc includes $\left\langle r^{2}\right\rangle_{0}=$ $\int d^{3} r r^{2} \rho_{0}(\vec{r}) / A$ with $\rho_{0}(\vec{r})$ being the g.s. density. The operator is determined by the curl of the nuclear current and so is vortical.

In this paper, we present additional arguments that, despite the PDR/TDR differences (e.g. irrotational vs vortical flow), the isoscalar PDR may rather be considered as a local manifestation of the general toroidal motion [3]. The collectivity of the TDR is inspected in detail. Like in the study [5], the analysis is done for ${ }^{208} \mathrm{~Pb}$ in the framework of the 1d RPA with the Skyrme force SLy6 [11].

\section{Discussion}

In Figure 1, the dipole strength functions are shown for the relevant resonances. The energy-dependent Lorentz smoothing [12] is used to roughly simulate the dissipation effects (coupling to complex configuration and continuum) beyond our RPA description. Panel (a) indicates the regions of PDR and giant dipole resonance (GDR). The PDR has two peaks at 7.5 and $10.3 \mathrm{MeV}$, which is in agreement with the previous calculations using relativistic mean field (RMF) [13] or Skyrme functional [3]. In the panels $(\mathrm{b}, \mathrm{c})$, the isoscalar TDR and its high-energy counterpart, the compression dipole resonance (CDR) $[14,15]$, are depicted. It is seen that the TDR lies at $6-9 \mathrm{MeV}$, i.e. just at the energy interval where the isoscalar PDR is located. Moreover the TDR energy almost coincides with the position of the PDR peak at 7.5 MeV. So the PDR and TDR are related. Also we see here a small fraction of the CDR.

Panel (b) demonstrates a considerable difference between RPA and unperturbed (1ph) TDR responses. The residual isoscalar interaction noticeably downshifts the strength and leads to the strong peak at 7.5 MeV. Actually this peak embraces two RPA states at 7.4 and $7.7 \mathrm{MeV}$. 


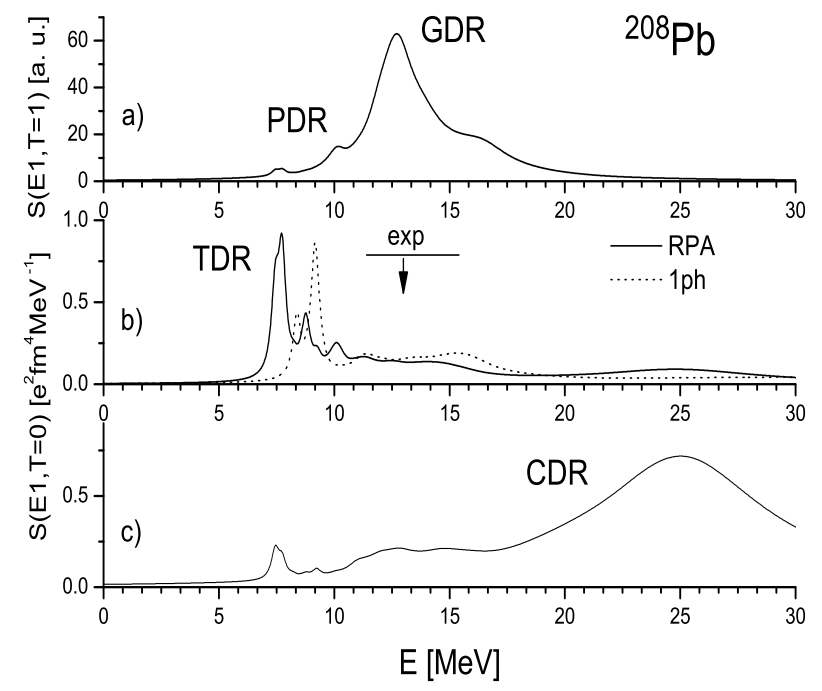

Figure 1. Calculated strength functions: (a) energy-weighted E1(T=1) GDR and PDR, (b) E1(T=0) TDR (RPA and unperturbed 1ph), (c) E1(T=0) CDR. In (b), the experimental width and energy of the ISGDR low-energy branch [9] are denoted.

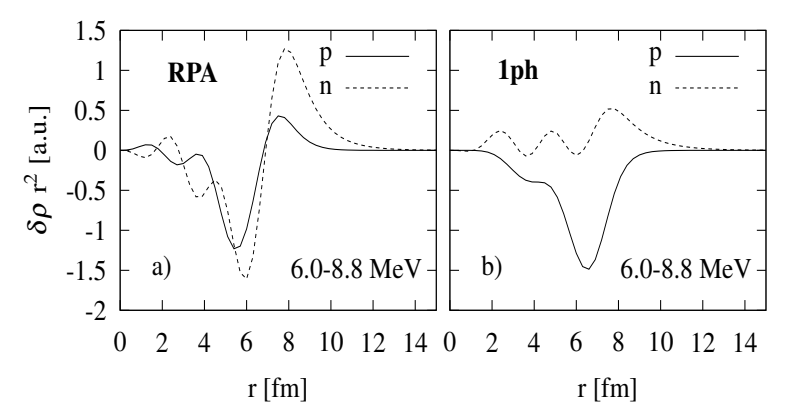

Figure 2. The calculated RPA (left) and unperturbed 1ph (right) $r^{2}$-weighted proton and neutron TD summed over the energy interval 6.0-8.8 $\mathrm{MeV}[5]$.

The states emerge from superposition of several $1 \mathrm{ph}$ components with maximal ones exhausting $67 \%$ and $23 \%$ of the norm, respectively. So the TDR seems to be collective.

Panel (b) also shows that the calculated TDR lies noticeably below the low-energy ISGDR branch observed in the $\left(\alpha, \alpha^{\prime}\right)$ reaction at $12-14 \mathrm{MeV}$ [9]. The same discrepancy takes place in most of the theoretical studies [1]. Following panel (c), perhaps not the TDR but the low-energy CDR bump was observed. Note also that the experiment [9] has inspected the excitation energy interval 8-35 MeV and so might lose the strong and narrow TM peak at 7$8 \mathrm{MeV}$. Therefore, additional experiments to search the isoscalar TDR are desirable.

Strength functions, being integral observables, are too rough for a thorough analysis of the subtle relation between TDR and PDR. They should be complemented by more detailed observables: transition densities (TD) and current transition densities (CTD) visualizing the coordinate dependence of the nuclear flow. In order to weaken the sensitivity of the results to individual dipole states and

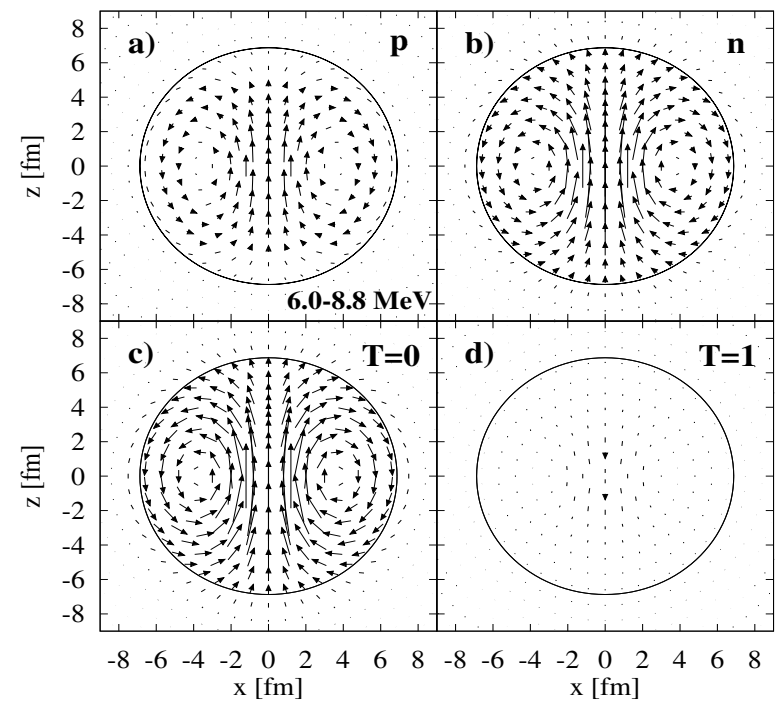

Figure 3. RPA proton (a), neutron (b), $\mathrm{T}=0$ (c), and $\mathrm{T}=1$ (d) CTD summed over the energy interval 6.0-8.8 MeV.

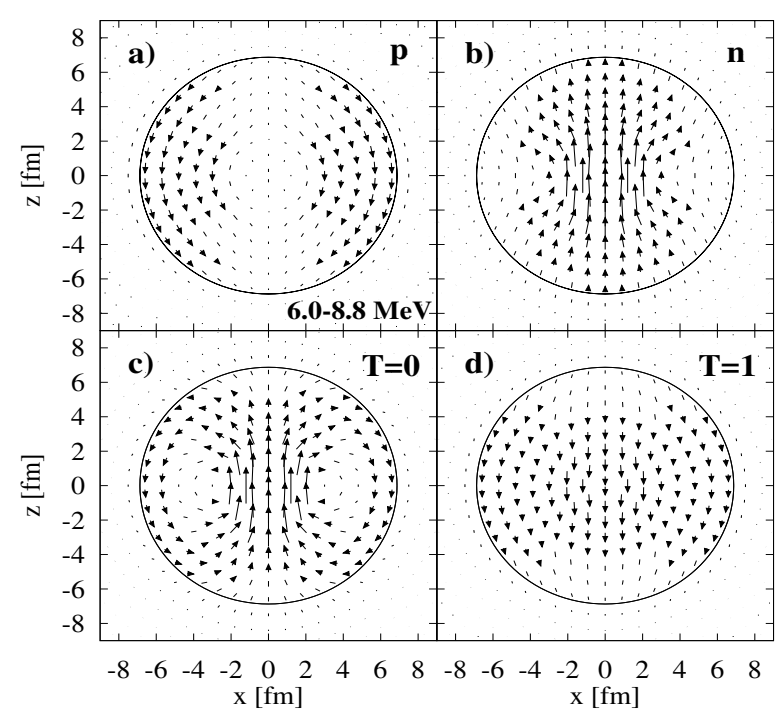

Figure 4. Unperturbed (1ph) proton (a), neutron (b), $\mathrm{T}=0$ (c), and $\mathrm{T}=1$ (d) CTD summed over the energy interval 6.0-8.8 MeV.

so to focus on the underlying gross features of flow, the TD/CTD strengths are summed over all RPA states in a properly chosen energy interval. This is done here for the interval 6.0-8.8 MeV embracing both $\mathrm{T}=0$ PDR and TDR. To circumvent the ambiguity in signs the RPA states, the $\mathrm{TD} / \mathrm{CTD}$ are calculated with the corresponding isoscalar weights, see [5] for details. In CTD the convection nuclear current is used.

The treatment of the PDR as oscillations of a neutron excess against the nuclear $N=Z$ core is often justified by the specific picture of the PDR transition densities where neutron and protons move by a similar manner in the nuclear interior while the neutron motion strongly dominates at the nuclear surface [1]. In Fig. 2(a), these are regions 4-7 fm and 7-11 fm, respectively. It is also seen (plot (b)) that the pygmy-like picture is lost in the summed $1 \mathrm{ph}$ TD. 


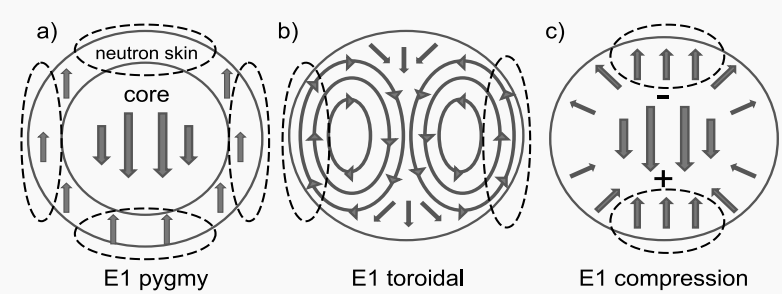

Figure 5. Schematic velocity fields for the PDR (a), TDR (b), and CDR (c) [5]. The driving field is directed along z-axis. In the plot (c), the compression (+) and decompression (-) regions are marked. The dashed ovals show similar local peripheral flows in the PDR and TDR/CDR.

However TD illustrates only radial motion while a thorough flow image needs an angular view as well. The latter is provided by the CTD given in Figs. 3 and 4 for the RPA and 1 ph cases, respectively. Fig. 3 shows that neutron and, in a less extent, proton motions exhibit a typical toroidal flow (to be compared to the schematic TDR view in Fig. 5(b)). Both motions are in phase and give altogether a strong isoscalar TDR while the isovector field is suppressed. So, following the current fields, the E1 RPA excitations at 6.0-8.8 MeV (location of T $=0$ PDR) are mainly of isoscalar vortical toroidal nature. A similar result for CTD was obtained earlier within the quasiparticlephonon model [16].

We also see clear signs of toroidal flow in unperturbed neutron and proton current fields depicted in Fig. 4. However, unlike the RPA case, the T=1 GDR-like field is comparable here in magnitude with the $\mathrm{T}=0$ toroidal field. This difference between RPA and $1 \mathrm{ph}$ cases, together with those in Figs. 1 and 2, allows to assign some collectivity to the $\mathrm{T}=0 \mathrm{TDR}$.

The natural question is how to conform the rather contradicting irrotational PDR and vortical TDR pictures. For this aim, it is instructive to consider schematic views [5] of the PDR, TDR, and CDR, given in Fig. 5. These idealized pictures indicate that PDR and CDR are indeed irrotational while the TDR is fully vortical. This is additionally confirmed by expressions for their velocities [10]: $\vec{v}_{\mathrm{PDR}, \mathrm{GDR}}=\vec{\nabla}\left(r Y_{1 \mu}\right), \vec{v}_{\mathrm{CDR}}=\vec{\nabla}\left(r^{3} Y_{1 \mu}\right)$, and $\vec{v}_{\mathrm{TDR}}=r^{2} \vec{Y}_{12 \mu}$. However, the peripheral motion of the neutron excess in the PDR is similar to the peripheral nucleon motions in the TDR (left/right sides) and CDR (up/bottom sides), as indicated in Fig. 5 by dashed ovals. In other words, despite the complete toroidal motion is vortical, its peripheral left and right parts look irrotational. Hence the principle difference between the irrotational PDR and vortical TDR becomes locally insignificant and the PDR can indeed be seen as a local manifestation of the TDR.

The next PDR/TDR difference is that the PDR exists only in nuclei with the neutron excess while the TDR is pertinent to all nuclei. However this also does not contradict our treatment since in nuclei with $\mathrm{N}>\mathrm{Z}$, the peripheral flow in TDR and CDR should be dominated by neutrons. Anyway our analysis should still be further checked by systematic calculations for PDR/TDR in different mass re- gions. It should be verified if indeed the PDR excitations always share the energy region with the TDR and exhibit the toroidal current fields. Though the coupling with complex configurations is essential in the PDR region [4], it should not affect much the summed current fields (as follows from the similarity of our fields to those from [16]).

Though E1 excitations at $6.0-8.8 \mathrm{MeV}$ seem to be mainly toroidal, they can also have minor contributions from other modes (CDR, GDR tail, individual 1ph configurations) which are not well visible in the current fields but follow from the analysis of the strength functions. So, most probably, these excitations are a complicated mixture of various coupled modes with their own doorway states. Depending on the experimental reaction, only the modes with proper doorway states are directly excited while others are involved only due to the coupling. Then, depending on the experimental probe, the dipole states can respond as the PDR in photoabsorption or T=0 TDR/CDR in $\left(\alpha, \alpha^{\prime}\right)$.

In summary, following our analysis, the isoscalar part of the PDR can be interpreted (fully or partly) as a manifestation of the toroidal resonance at the nuclear surface. The TDR demonstrates some collectivity.

The work was partly supported by the DFG grant RE 322/14-1, Heisenberg-Landau (Germany-BLTP JINR), and Votruba-Blokhincev (Czech Republic-BLTP JINR) grants. P.-G.R. is grateful for the BMBF support under Contracts No, 06 DD 9052D and 06 ER 9063. The support of the Czech Science Foundation (P203-13-07117S) is appreciated.

\section{References}

[1] N. Paar, D. Vretenar, E. Khan, and G. Colo, Rep. Prog. Phys. 70, 691 (2007)

[2] D. Savran, T. Aumann, and A. Zilges, Prog. Part. Nucl. Phys. 70, 210 (2013).

[3] P.-G. Reinhard and W. Nazarewicz, Phys. Rev. C 87, 014324 (2013)

[4] J. Endres et al., Phys. Rev. Lett. 105, 212503 (2010)

[5] A. Repko, P.-G. Reinhard, V. O. Nesterenko, and J. Kvasil, Phys. Rev. C87, 024305 (2013)

[6] V.M. Dubovik and A.A. Cheshkov, Sov. J. Part. Nucl. 5, 318 (1975)

[7] S.F. Semenko, Sov. J. Nucl. Phys. 34, 356 (1981)

[8] P.-G. Reinhard, V.O. Nesterenko, A. Repko, and J. Kvasil, Phys. Rev. C89, 024321 (2014)

[9] M. Uchida et al., Phys. Rev. C69, 051301(R) (2004)

[10] J. Kvasil, V.O. Nesterenko, W. Kleinig, P.-G. Reinhard, and P.Vesely, Phys. Rev. C84, 034303 (2011)

[11] E. Chabanat et al., Nucl. Phys. A635, 231 (1998)

[12] J. Kvasil, V.O. Nesterenko, W. Kleinig, D. Božík, and P.-G. Reinard, Int. J. Mod. Phys. E20 281 (2011)

[13] D. Vretenar, N. Paar, P. Ring, and G.A. Lalazissis, Phys. Rev. C63, 047301 (2001).

[14] M.N. Harakeh et al., Phys. Rev. Lett. 38, 676 (1977)

[15] S. Stringari, Phys. Lett. B108, 232 (1982)

[16] N. Ryezayeva et al., Phys. Rev. Lett. 89, 272502 (2002) 
